\title{
Evidence-Based Complementary Medicine - a Need for the Future
}

On April 6-8, 2000, an international congress on clinical research and quality management in complementary medicine will be held in Munich, Germany. The abstracts of the presentations are included in this issue of Forschende Komplementärmedizin.

The congress is organized by The Centre for Complementary Medicine Research, Department Internal Medicine II, Technical University of Munich, in collaboration with The Centre for Alternative Medicine Research and Education, Beth Israel Deaconess Medical Center, Harvard Medical School, Boston, MA.

Although complementary therapies are widely used throughout the world, there is a paucity of clinically relevant research and quality assurance in this area.

In the last decade, the amount of research activities has steadily grown in many countries. Especially in the USA the interest in complementary medicine has dramatically increased. Still, a research infrastructure for the vast field is developing only slowly. Researchers are sometimes isolated and studies are published in a plethora of journals. Common platforms rarely exist.

In a time of evidence-based medicine, growing needs to rationing of health care services and huge interest of consumers it seems timely that those performing clinical research and quality management in the field start to build a forum.
As congress president I really hope that the event will be the first of a series of regular conferences at changing places all over the world. A number of leading international experts should help to make this first conference a successful event.

As clinical research in this area is still in relative infancy, the discussion on adequate methodologies will be a central topic at this conference. Without the presentation of new original research, however, the discussion of methodology would be too abstract. Therefore, researchers were encouraged to submit their clinical research studies for presentation.

Beside the question 'does it work?' it is also useful to know 'who is providing the method with which kind of quality?'. These quality assurance-oriented questions will also be discussed.

I hope many of the readers of Forschende Komplementärmedizin are able to attend and will share a good time with us in order to build up a growing scientific community.

Dieter Melchart, München 